\title{
Stochastic responses of
}

\section{multi-degree-of-freedom uncertain hysteretic systems}

\author{
Yimin Zhang* \\ School of Mechanical Engineering and Automation, Northeastern University, Shenyang, 110004, China \\ Received 9 February 2010 \\ Revised 2 June 2010
}

\begin{abstract}
On the basis of the Bouc-Wen hysteretic model, the effective numerical method for the response of nonlinear multidegree-of-freedom (MDOF) stochastic hysteretic systems is presented using second moment method. Using this method, the mean values, variances and covariances are computed. The Monte Carlo simulation is applied to validate the method. The results obtained by the two methods are contrasted, and the solutions of the method in this paper agreed very well with the Monte Carlo simulation. It has solved the random response of nonlinear stochastic vibration systems which is caused by the stochastic hysteretic loop itself.
\end{abstract}

\section{Introduction}

Hysteretic system is one of the most typical nonlinear systems. Many engineering structural systems under dynamic loading usually exhibit hysteretic behavior, especially when the response becomes inelastic. A hysteretic loop produces between hysteretic restoring force and displacement under periodic movement. The effect exhibits the change of stiffness and energy. Because the restoring force of a hysteretic system depends not only on the instantaneous displacement, but also on its past history, analytical modeling and solution of such a system under random excitation has been an interesting and challenging subject. The hereditary nature of the restoring force of an inelastic system indicates that the force can no longer be described by an algebraic function of the instantaneous displacement and velocity. The research on hysteretic system concentrated on three aspects [1]: The first is the hysteretic restoring force model. Many different hysteretic restoring force models have been developed according to different engineering structural system needs, for example: Bilinear model [2], Ramberg-Osgood model [3], Differential equation models [4,5], etc. The second is the response of hysteretic system under random excitation. According to the hysteretic restoring force models, the response of such hysteretic system under random excitation had been solved. The main methods include the K-B approximation for bilinear systems [2], Semiempirical method for elastic-plastic systems [6,7], Equivalent linearization method for smooth systems [8-10], and Markov vector and Fokker-Plank equation method [11,12], etc. The last one is hysteretic system parameter identification. Generally, the response of system and energy dissipation and degrading can be analyzed after the hysteretic nonlinear relation of displacement and load of system are known. A simple technique based on a least square error minimization has been developed for the smooth hysteretic models. To hysteretic system, it is familiar that one model is firstly supposed basing on experience and such the parameters are recognized using vary means. Except above-mentioned measures, the methods of non-parameter recognizing are effective [13-15].

\footnotetext{
*Corresponding author. E-mail: zhangymneu@sohu.com.
} 
For a last decade, the response of hysteretic system under random excitation or with stochastic parameters has been further investigated. A method was presented to capture the drift of some hysteretic systems under a zero-mean, broad-band and stationary-random load in [16]. The stochastic response of a Preisach hysteretic system applied by a white noise process was studied and an approximate solution method was proposed in [17]. The hysteretic response of a high number of DOF 3D frames under stochastic load has been investigated in [18]. The modeling and analysis for the hysteretic nonlinear system which is applied by deterministic excitation and is with the stochastic parameters have been developed in [19]. To solve the response of a SDF Preisach hysteretic system which is with nonlocal memory and applied by the stationary Gaussian, a new stochastic averaging technique was proposed in [20].

According to the above works, it could be seen that Bouc-Wen differential coefficient model has been used abroad to formulate the hysteretic restoring force, the response of single-degree-of-freedom or multi-degree-of-freedom hysteretic system under random excitation according to different requires could be solved by the existed methods and there have been some fruits which could identify the hysteretic system parameter.

However, the random response of nonlinear stochastic vibration system caused by the hysteretic loop that is random itself has not been solved. The semi-continuous medium in engineering mechanism stands out especially, because of the discrete characteristic of material, which leads to the nonlinear vibration model of random hysteretic system. A lot of engineering experiments have already proved the random discrete characteristic that lies in hysteretic loop itself. So it is very necessary to study the random vibration of nonlinear systems that caused by uncertain hysteretic loop.

In this paper, the stochastic hysteretic model is considered. On the basis of the Bouc-Wen hysteretic model, the second moment method is applied to get the effective numerical method for the response of multi-degree-of-freedom stochastic hysteretic system that is caused by uncertain hysteretic loop itself. The example is given according to the method and Monte Carlo simulation. The results that are obtained by the two methods are contrasted, and they are consistent with each other very well, so it is correct to use the method to solve the response of stochastic hysteretic system. It has solved the random response of nonlinear stochastic vibration system that is caused by the stochastic hysteretic loop itself.

\section{Random hysteretic system}

It is known that the nonlinear motion equations of a structural vibration are

$$
\mathbf{g}(\ddot{\mathbf{x}}, \dot{\mathbf{x}}, \mathbf{x}, \mathbf{b})=\mathbf{f}(\mathbf{b}, t)
$$

with initial conditions

$$
\mathbf{x}(0)=\mathbf{x}_{0}, \dot{\mathbf{x}}(0)=\dot{\mathbf{x}}_{0}
$$

where $g, x=(y, z)$ and $f$ are the nonlinear function, displacement and external force respectively and a superscript dot represents the time $(t)$ derivative. The probabilistic effects are described through the random parameter vector $b=\left(b_{i}\right)_{s \times 1}$ of order $s$, which can include the probabilistic distributions of all discretized random variable properties.

The smooth hysteretic model was proposed initially by Bouc and extended by Wen. A function of the time history of $y, z$ is related to $x$ through the following first-order nonlinear differential equation.

$$
\dot{z}_{i}(t)=-\gamma_{i}\left|\dot{y}_{i}\right| z_{i}\left|z_{i}\right|^{n-1}-\beta_{i} \dot{y}_{i}\left|z_{i}\right|^{n}+A_{i} \dot{y}_{i}
$$

where $\gamma_{i}, \beta_{i}, A_{i}$, and $n$ are parameters. It has been shown that a hysteretic relationship exists between $z$ and $y$ and one can construct a variety of restoring forces, such as softening hardening, narrow or wide-band systems. Parameters $\gamma_{i}$ and $\beta_{i}$ control the shape of the hysteresis loop, $A_{i}$ the restoring force amplitude, and $n$ the smoothness of the transition from elastic to plastic response, e.g., a large value of $n$ corresponds to an almost elasto-plastic system. 


\section{Dynamic responses of uncertain hysteretic system}

Suppose $B(s \times t)$ and $A(p \times 1)$ are matrices of random variables related by the transformation $A=A(B)$. Then the second order Taylor expansion of $A^{[21]}$ about a nominal value $\overline{\mathbf{B}}$ of $B$ is given

$$
\begin{aligned}
\mathbf{A}(\mathbf{B}) & =\mathbf{A}(\overline{\mathbf{B}})+\left.\frac{\partial \mathbf{A}}{\partial(\operatorname{cs} \mathbf{B})^{T}}\right|_{\mathbf{B}=\overline{\mathbf{B}}} \mathrm{d}[\operatorname{cs}(\mathbf{B})] \\
& +\left.\frac{1}{2} \frac{\partial^{2} \mathbf{A}}{\partial(\operatorname{cs} \mathbf{B})^{\mathrm{T}}}\right|_{\mathbf{B}=\overline{\mathbf{B}}}\{\mathrm{d}[\operatorname{cs}(\mathbf{B})]\}^{[2]}
\end{aligned}
$$

where $\operatorname{cs}(B)$ is the column representation of $B, \mathrm{~d}[\operatorname{cs}(\mathbf{B})]^{[2]}=\mathrm{d}[\operatorname{cs}(\mathbf{B})] \otimes \mathrm{d}[\operatorname{cs}(\mathbf{B})]$ is the second order Kronecker power of $\mathrm{d}[\operatorname{cs}(B)]=\varepsilon \Delta \operatorname{cs}(B), \varepsilon$ is a book keeping parameter, $\Delta$ is a deference operator defined by $\Delta \operatorname{cs}(B)=$ $[\operatorname{cs}(B)-\operatorname{cs}(\overline{\mathbf{B}})]$, and $\otimes$ represents Kronecker product.

To derive the random matrix equations for nonlinear structural dynamics, the following notations are used. For a given vector-valued and matrix-valued function $A(B)$ and a small parameter $\varepsilon: \overline{\mathbf{B}}=\mathrm{E}(\mathbf{B})$ is the mean value matrix of $B$, i.e., the expectation matrix $\mathrm{E}$ ( ) of $B . \mathrm{d} \mathbf{B}=\varepsilon \Delta \mathbf{B}=\varepsilon(\mathbf{B}-\overline{\mathbf{B}})$ is first-order variation matrix of $B$ about $\overline{\mathbf{B}}$. $[\mathrm{d} \mathbf{B}]^{[2]}=\varepsilon^{2} \Delta \mathbf{B}^{[2]}=\varepsilon^{2}(\mathbf{B}-\overline{\mathbf{B}})^{[2]}$ is second-order variation matrix of about $\overline{\mathbf{B}}$ and $\overline{\mathbf{A}}=\mathbf{A}(\overline{\mathbf{B}})$ is the value of $A$ evaluated at $\overline{\mathbf{B}}$

The expressions of first order moment, second order moment have following forms [22]

$$
\begin{aligned}
& \mathrm{E}[\mathbf{A}(\mathbf{B})]=\mathbf{A}[\mathrm{E}(\mathbf{B})]+\frac{1}{2} \frac{\partial^{2} \mathbf{A}}{\partial(\operatorname{cs} \mathbf{B})^{\mathrm{T}_{2}}}[\operatorname{Var}(\operatorname{csB})] \\
& \operatorname{Var}[\mathbf{A}(\mathbf{B})]=\left[\frac{\partial \mathbf{A}}{\partial(\operatorname{csB})^{T}}\right]^{[2]} \operatorname{Var}(\operatorname{csB})
\end{aligned}
$$

where $\mathrm{E}()$ is the mean value matrix, $\operatorname{Var}()$ is the variance matrix which includes variance and covariances. According to the above expressions, the mean values are accurate to the second order, the variances and covariances are accurate to the first order.

The matrices of the both sides of Eq. (1) are expanded about $\overline{\mathbf{b}}$ via Taylor series

$$
\begin{aligned}
\mathbf{g} & =\overline{\mathbf{g}} \\
& +\left[\frac{\partial \overline{\mathbf{g}}}{\partial \mathbf{b}^{T}}+\frac{\partial \overline{\mathbf{g}}}{\partial \ddot{\mathbf{x}}^{T}} \frac{\partial \overline{\overline{\mathbf{x}}}}{\partial \mathbf{b}^{T}}+\frac{\partial \overline{\mathbf{g}}}{\partial \dot{\mathbf{x}}^{T}} \frac{\partial \overline{\dot{\mathbf{x}}}}{\partial \mathbf{b}^{T}}+\frac{\partial \overline{\mathbf{g}}}{\partial \mathbf{x}^{T}} \frac{\partial \overline{\mathbf{x}}}{\partial \mathbf{b}^{T}}\right] \mathrm{d} \mathbf{b} \\
& +\frac{1}{2}\left[\frac{\partial^{2} \overline{\mathbf{g}}}{\partial \mathbf{b}^{T}}+\frac{\partial^{2} \overline{\mathbf{g}}}{\partial \mathbf{b}^{T} \partial \ddot{\mathbf{x}}^{T}}\left(\frac{\partial \overline{\mathbf{x}}}{\partial \mathbf{b}^{T}} \otimes \mathbf{I}_{s}\right)\right. \\
& +\frac{\partial^{2} \overline{\mathbf{g}}}{\partial \mathbf{b}^{T} \partial \dot{\mathbf{x}}^{T}}\left(\frac{\partial \overline{\dot{\mathbf{x}}}}{\partial \mathbf{b}^{T}} \otimes \mathbf{I}_{s}\right)+\frac{\partial^{2} \overline{\mathbf{g}}}{\partial \mathbf{b}^{T} \partial \mathbf{x}^{T}}\left(\frac{\partial \overline{\mathbf{x}}}{\partial \mathbf{b}^{T}} \otimes \mathbf{I}_{s}\right) \\
& +\frac{\partial^{2} \overline{\mathbf{g}}}{\partial \ddot{\mathbf{x}}^{T} \partial \mathbf{b}^{T}}\left(\mathbf{I}_{s} \otimes \frac{\partial \overline{\mathbf{x}}}{\partial \mathbf{b}^{T}}\right)+\frac{\partial^{2} \overline{\mathbf{g}}}{\partial \dot{\mathbf{x}}^{T} \partial \mathbf{b}^{T}}\left(\mathbf{I}_{s} \otimes \frac{\partial \overline{\dot{\mathbf{x}}}}{\partial \mathbf{b}^{T}}\right) \\
& +\frac{\partial^{2} \overline{\mathbf{g}}}{\partial \mathbf{x}^{T} \partial \mathbf{b}^{T}}\left(\mathbf{I}_{\mathbf{s}} \otimes \frac{\partial \overline{\mathbf{x}}}{\partial \mathbf{b}^{T}}\right)+\frac{\partial \overline{\mathbf{g}}}{\partial \ddot{\mathbf{x}}^{T}} \frac{\partial^{2} \overline{\overline{\mathbf{x}}}}{\partial \mathbf{b}^{T} 2} \\
& \left.+\frac{\partial \overline{\mathbf{g}}}{\partial \dot{\mathbf{x}}^{T}} \frac{\partial^{2} \overline{\mathbf{x}}}{\partial \mathbf{b}^{T}}+\frac{\partial \overline{\mathbf{g}}}{\partial \mathbf{x}^{T}} \frac{\partial^{2} \overline{\mathbf{x}}}{\partial \mathbf{b}^{T} 2}\right](\mathrm{db})^{[2]} \\
\mathbf{f} & =\overline{\mathbf{f}}+\frac{\partial \overline{\mathbf{f}}}{\partial \mathbf{b}^{T}} \mathrm{~d} \mathbf{b}+\frac{1}{2} \frac{\partial^{2} \overline{\mathbf{f}}}{\partial \mathbf{b}^{T} 2}(\mathrm{db})^{[2]}
\end{aligned}
$$


Substituting Eqs (7)-(8) into Eq. (1) and equating similar order terms, one has the zeroth-order, first-order, secondorder equations corresponding to Eq.(1):

Zeroth order

$$
\overline{\mathbf{g}}=\overline{\mathbf{f}}
$$

First order ( $\varepsilon$ terms)

$$
\frac{\partial \overline{\mathbf{g}}}{\partial \ddot{\mathbf{x}}^{T}} \ddot{\mathbf{x}}_{1}+\frac{\partial \overline{\mathbf{g}}}{\partial \dot{\mathbf{x}}^{T}} \dot{\mathbf{x}}_{1}+\frac{\partial \overline{\mathbf{g}}}{\partial \mathbf{x}^{T}} \mathbf{x}_{1}=\mathbf{f}_{1}
$$

where

$$
\begin{aligned}
& \ddot{\mathbf{x}}_{1}=\frac{\partial \overline{\overline{\mathbf{x}}}}{\partial \mathbf{b}^{T}}(\mathbf{b}-\overline{\mathbf{b}}) \\
& \dot{\mathbf{x}}_{1}=\frac{\partial \overline{\dot{\mathbf{x}}}}{\partial \mathbf{b}^{T}}(\mathbf{b}-\overline{\mathbf{b}}) \\
& \mathbf{x}_{1}=\frac{\partial \overline{\mathbf{x}}}{\partial \mathbf{b}^{T}}(\mathbf{b}-\overline{\mathbf{b}}) \\
& \mathbf{f}_{1}=\left[\frac{\partial \overline{\mathbf{f}}}{\partial \mathbf{b}^{T}}-\frac{\partial \overline{\mathbf{g}}}{\partial \mathbf{b}^{T}}\right](\mathbf{b}-\overline{\mathbf{b}})
\end{aligned}
$$

Second order $\left(\varepsilon^{2}\right.$ terms $)$

$$
\frac{\partial \overline{\mathbf{g}}}{\partial \ddot{\mathbf{x}}^{T}} \overline{\mathbf{x}}_{2}+\frac{\partial \overline{\mathbf{g}}}{\partial \dot{\mathbf{x}}^{T}} \overline{\dot{\mathbf{x}}}_{2}+\frac{\partial \overline{\mathbf{g}}}{\partial \mathbf{x}^{T}} \overline{\mathbf{x}}_{2}=\overline{\mathbf{f}}_{2}
$$

where

$$
\begin{aligned}
& \overline{\ddot{\mathbf{x}}}_{2}=\frac{1}{2} \frac{\partial^{2} \overline{\ddot{\mathbf{x}}}}{\partial \mathbf{b}^{\mathrm{T}} 2} \operatorname{Var}(\mathbf{b}) \\
& \overline{\dot{\mathbf{x}}}_{2}=\frac{1}{2} \frac{\partial^{2} \overline{\dot{\mathbf{x}}}}{\partial \mathbf{b}^{\mathrm{T} 2}} \operatorname{Var}(\mathbf{b}) \\
& \overline{\mathbf{x}}_{2}=\frac{1}{2} \frac{\partial^{2} \overline{\mathbf{x}}}{\partial \mathbf{b}^{\mathrm{T} 2}} \operatorname{Var}(\mathbf{b}) \\
& \overline{\mathbf{f}}_{2}=\frac{1}{2}\left[\frac{\partial^{2} \overline{\mathbf{f}}}{\partial \mathbf{b}^{T} 2}-\frac{\partial^{2} \overline{\mathbf{g}}}{\partial \mathbf{b}^{T} 2}-\frac{\partial^{2} \overline{\mathbf{g}}}{\partial \mathbf{b}^{T} \partial \ddot{\mathbf{x}}^{T}}\left(\frac{\partial \overline{\overline{\mathbf{x}}}}{\partial \mathbf{b}^{T}} \otimes \mathbf{I}_{s}\right)\right. \\
& -\frac{\partial^{2} \overline{\mathbf{g}}}{\partial \mathbf{b}^{T} \partial \dot{\mathbf{x}}^{T}}\left(\frac{\partial \overline{\dot{\mathbf{x}}}}{\partial \mathbf{b}^{T}} \otimes \mathbf{I}_{s}\right)-\frac{\partial^{2} \overline{\mathbf{g}}}{\partial \mathbf{b}^{T} \partial \mathbf{x}^{T}}\left(\frac{\partial \overline{\mathbf{x}}}{\partial \mathbf{b}^{T}} \otimes \mathbf{I}_{s}\right) \\
& -\frac{\partial^{2} \overline{\mathbf{g}}}{\partial \ddot{\mathbf{x}}^{T} \partial \mathbf{b}^{T}}\left(\mathbf{I}_{s} \otimes \frac{\partial \overline{\ddot{\mathbf{x}}}}{\partial \mathbf{b}^{T}}\right)-\frac{\partial^{2} \overline{\mathbf{g}}}{\partial \dot{\mathbf{x}}^{T} \partial \mathbf{b}^{T}}\left(\mathbf{I}_{s} \otimes \frac{\partial \overline{\dot{\mathbf{x}}}}{\partial \mathbf{b}^{T}}\right) \\
& \left.-\frac{\partial^{2} \overline{\mathbf{g}}}{\partial \mathbf{x}^{T} \partial \mathbf{b}^{T}}\left(\mathbf{I}_{\mathbf{S}} \otimes \frac{\partial \overline{\mathbf{x}}}{\partial \mathbf{b}^{T}}\right)\right] \operatorname{Var}(\mathbf{b})
\end{aligned}
$$

Once $\overline{\ddot{\mathbf{x}}}, \overline{\dot{\mathbf{x}}}, \overline{\mathbf{x}}, \overline{\mathbf{x}}_{2}, \overline{\dot{\mathbf{x}}}_{2}$, and $\overline{\mathbf{x}}_{2}$ are obtained, $\ddot{\mathbf{x}}_{1}, \dot{\mathbf{x}}_{1}$ and $\mathbf{x}_{1}$ can be determined. The mean value, variance matrices of the responses can then be computed.

According to the second-moment technique, the mean value, variance of the responses are represented as

$$
\begin{aligned}
& \mathrm{E}(\ddot{\mathbf{x}})=\overline{\ddot{\mathbf{x}}}+\overline{\ddot{\mathbf{x}}}_{2} \\
& \mathrm{E}(\overline{\dot{\mathbf{x}}})=\overline{\dot{\mathbf{x}}}+\overline{\mathbf{x}}_{2}
\end{aligned}
$$




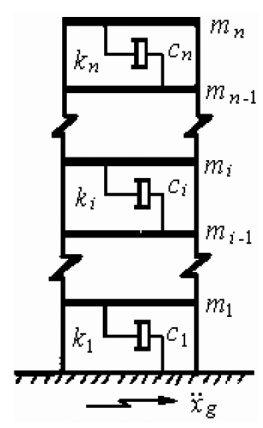

Fig. 1. System model.

$$
\begin{aligned}
& \mathrm{E}(\mathbf{x})=\overline{\mathbf{x}}+\overline{\mathbf{x}}_{2} \\
& \operatorname{Var}(\ddot{\mathbf{x}})=\mathrm{E}\left(\ddot{\mathbf{x}}_{1} \otimes \ddot{\mathbf{x}}_{1}\right)=\left[\frac{\partial \overline{\overline{\mathbf{x}}}}{\partial \mathbf{b}^{T}}\right]^{[2]} \operatorname{Var}(\mathbf{b}) \\
& \operatorname{Var}(\dot{\mathbf{x}})=\mathrm{E}\left(\dot{\mathbf{x}}_{1} \otimes \dot{\mathbf{x}}_{1}\right)=\left[\frac{\partial \overline{\dot{\mathbf{x}}}}{\partial \mathbf{b}^{T}}\right]^{[2]} \operatorname{Var}(\mathbf{b}) \\
& \operatorname{Var}(\mathbf{x})=\mathrm{E}\left(\mathbf{x}_{1} \otimes \mathbf{x}_{1}\right)=\left[\frac{\partial \overline{\mathbf{x}}}{\partial \mathbf{b}^{T}}\right]^{[2]} \operatorname{Var}(\mathbf{b})
\end{aligned}
$$

In order to get the derivations $\frac{\partial \overline{\ddot{\mathbf{x}}}}{\partial \mathbf{b}^{T}}, \frac{\partial \overline{\dot{\mathbf{x}}}}{\partial \mathbf{b}^{T}}, \frac{\partial \overline{\mathbf{x}}}{\partial \mathbf{b}^{T}}$ substituting Eqs (11)-(14) into Eq.(10) and equating similar terms, one obtains the sensitivity equations

$$
\begin{aligned}
& \frac{\partial \mathbf{g}}{\partial \ddot{\mathbf{x}}^{T}} \frac{\partial \overline{\ddot{\mathbf{x}}}}{\partial b_{i}}+\frac{\partial \mathbf{g}}{\partial \dot{\mathbf{x}}^{T}} \frac{\partial \overline{\dot{\mathbf{x}}}}{\partial b_{i}} \\
& +\frac{\partial \mathbf{g}}{\partial \mathbf{x}^{T}} \frac{\partial \overline{\mathbf{x}}}{\partial b_{i}}=\frac{\partial \overline{\mathbf{f}}}{\partial b_{i}}(i=1,2, \cdots, s)
\end{aligned}
$$

Thus, the derivation matrices $\frac{\partial \overline{\ddot{\mathbf{x}}}}{\partial \mathbf{b}^{T}}, \frac{\partial \overline{\dot{\mathbf{x}}}}{\partial \mathbf{b}^{T}}, \frac{\partial \overline{\mathbf{x}}}{\partial \mathbf{b}^{T}}$ can be found from

$$
\begin{aligned}
\frac{\partial \overline{\dot{\mathbf{x}}}}{\partial \mathbf{b}^{T}} & =\left[\frac{\partial \overline{\dot{\mathbf{x}}}}{\partial b_{1}} \frac{\partial \overline{\dot{\mathbf{x}}}}{\partial b_{2}} \cdots \frac{\partial \overline{\dot{\mathbf{x}}}}{\partial b_{s}}\right] \\
\frac{\partial \overline{\dot{\mathbf{x}}}}{\partial \mathbf{b}^{T}} & =\left[\frac{\partial \overline{\dot{\mathbf{x}}}}{\partial b_{1}} \frac{\partial \overline{\dot{\mathbf{x}}}}{\partial b_{2}} \cdots \frac{\partial \overline{\dot{\mathbf{x}}}}{\partial b_{s}}\right] \\
\frac{\partial \overline{\mathbf{x}}}{\partial \mathbf{b}^{T}} & =\left[\frac{\partial \overline{\mathbf{x}}}{\partial b_{1}} \frac{\partial \overline{\mathbf{x}}}{\partial b_{2}} \cdots \frac{\partial \overline{\mathbf{x}}}{\partial b_{s}}\right]
\end{aligned}
$$

Substituting Eqs (27)-(29) into Eqs (23)-(25), one obtains the variance matrices $\operatorname{Var}(\ddot{\mathbf{x}})$, $\operatorname{Var}(\dot{\mathbf{x}})$ and $\operatorname{Var}(\mathbf{x})$.

\section{Numerical example}

Soil as a construction material exhibits complicated behavior under loading, which can be highly nonlinear and hysteretic. Under earthquake excitation, the potential hazards caused by soil failure include liquefaction and foundation failure of a structure. To evaluate soil and structure performance under earthquake loadings, such nonlinear behavior of soil needs to be taken into consideration [23]. The system model is shown in Fig. 1. 


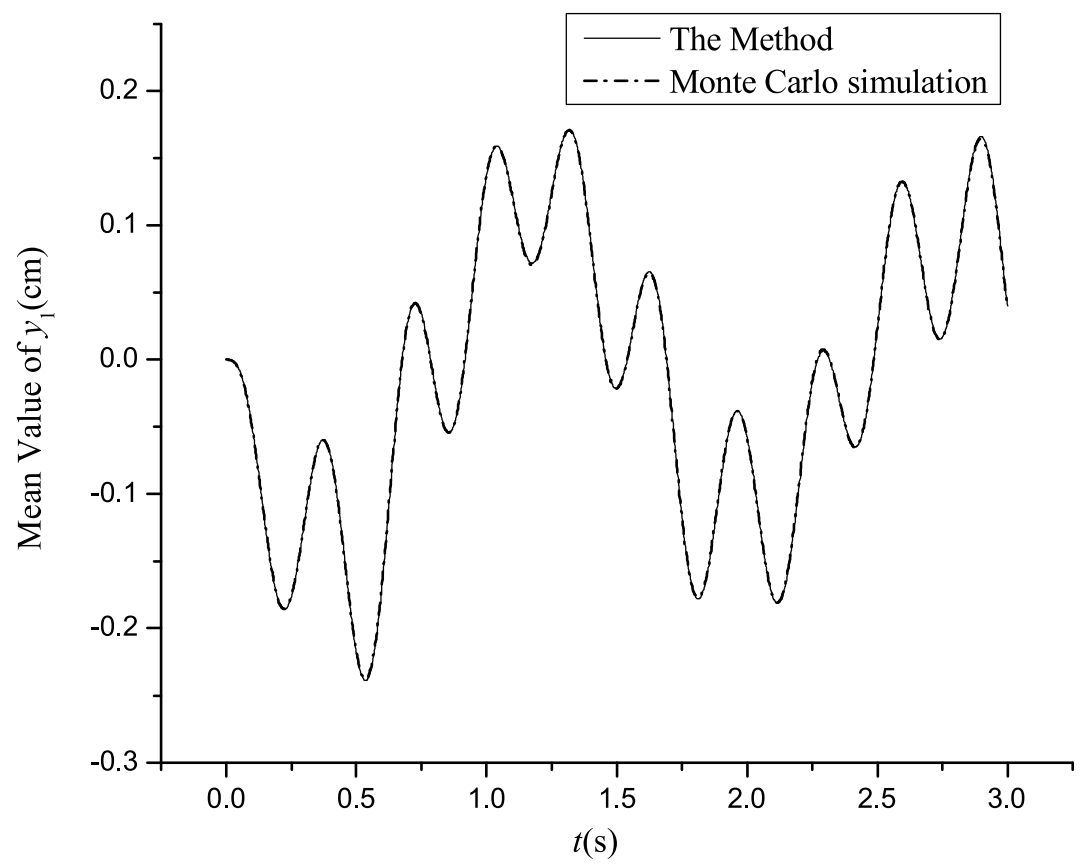

Fig. 2. The mean value curve of the displacement $y_{1}$.

$$
\left\{\begin{array}{c}
m_{i} \ddot{y}_{i}+c_{i} \dot{u}_{i}-c_{i+1} \dot{u}_{i+1}+V_{i}\left(u_{i}, z_{i}\right) \\
\quad-V_{i+1}\left(u_{i+1}, z_{i+1}\right)=-m_{i} \ddot{x}_{g} \\
\dot{z}_{i}=-\gamma_{i}\left|\dot{u}_{i}\right| z_{i}\left|z_{i}\right|^{n-1}-\beta_{i} \dot{u}_{i}\left|z_{i}\right|^{n}+A_{i} \dot{u}_{i}
\end{array}\right.
$$

The case of $n=1$ is considered, the smooth hysteretic model is described by

$$
\left\{\begin{array}{c}
m_{i} \ddot{y}_{i}+c_{i} \dot{u}_{i}-c_{i+1} \dot{u}_{i+1}+\alpha_{i} k_{i} u_{i} \\
\quad+\left(1-\alpha_{i}\right) k_{i} z_{i}-\left(1-\alpha_{i}\right) k_{i} z_{i-1} \\
\quad-\alpha_{i+1} k_{i+1} u_{i+1}-\left(1-\alpha_{i+1}\right) k_{i+1} z_{i+1} \\
\quad+\left(1-\alpha_{i+1}\right) k_{i+1} z_{i}=-m_{i} \ddot{x}_{g} \\
\dot{z}_{i}=-\gamma_{i}\left|\dot{u}_{i}\right| z_{i}-\beta_{i} \dot{u}_{i}\left|z_{i}\right|+A_{i} \dot{u}_{i}
\end{array}\right.
$$

To include the effects of soil-structure interaction, a two stories structural system model is given. The first two moments of the random parameters $\gamma_{i}, \beta_{i}, A_{i}$ are as follow respectively.

$$
\begin{aligned}
& \mathrm{E}\left(\gamma_{1}\right)=0.1, \quad \sigma_{\gamma_{1}}=0.005 \times 0.1, \\
& \mathrm{E}\left(\gamma_{2}\right)=0.5, \quad \sigma_{\gamma_{2}}=0.005 \times 0.5, \\
& \mathrm{E}\left(\beta_{1}\right)=0.9, \quad \sigma_{\beta_{1}}=0.005 \times 0.9, \\
& \mathrm{E}\left(\beta_{2}\right)=0.4, \quad \sigma_{\beta_{2}}=0.005 \times 0.4, \\
& \mathrm{E}\left(A_{1}\right)=1.0, \quad \sigma_{A_{1}}=0.005 \times 1.0, \\
& \mathrm{E}\left(A_{2}\right)=1.5, \quad \sigma_{A_{2}}=0.005 \times 1.5
\end{aligned}
$$

The deterministic masses, dampings, initial stiffness and external acceleration are $m_{1}=m_{2}=2.0(\mathrm{~kg}), c_{1}=c_{2}=$ $1.0(\mathrm{~N} \cdot \mathrm{s} / \mathrm{cm}), k_{1}=k_{2}=100(\mathrm{~N} / \mathrm{cm})$ and $\ddot{x}_{g}=20 \sin 20 \mathrm{t}\left(\mathrm{cm} / \mathrm{s}^{2}\right)$, respectively. The post to preyielding stiffness ratios $\alpha_{1}=\alpha_{2}=1.0 / 21.0$. The random parameter vector is $\mathbf{b}=\left\{\gamma_{1} \gamma_{2} \beta_{1} \beta_{2} A_{1} A_{2}\right\}^{T}$.

Each order equations derived earlier are solved by the Runge-Kutta method. The mean amplitudes $y_{1}$ and $y_{2}$ is depicted in Figs 2-3. For the variances of $y_{1}$ and $y_{2}$ the solution are plotted in Figs 4-5 and for the covariance of $y_{1}$ and $y_{2}$ the solution are plotted in Fig. 6. 


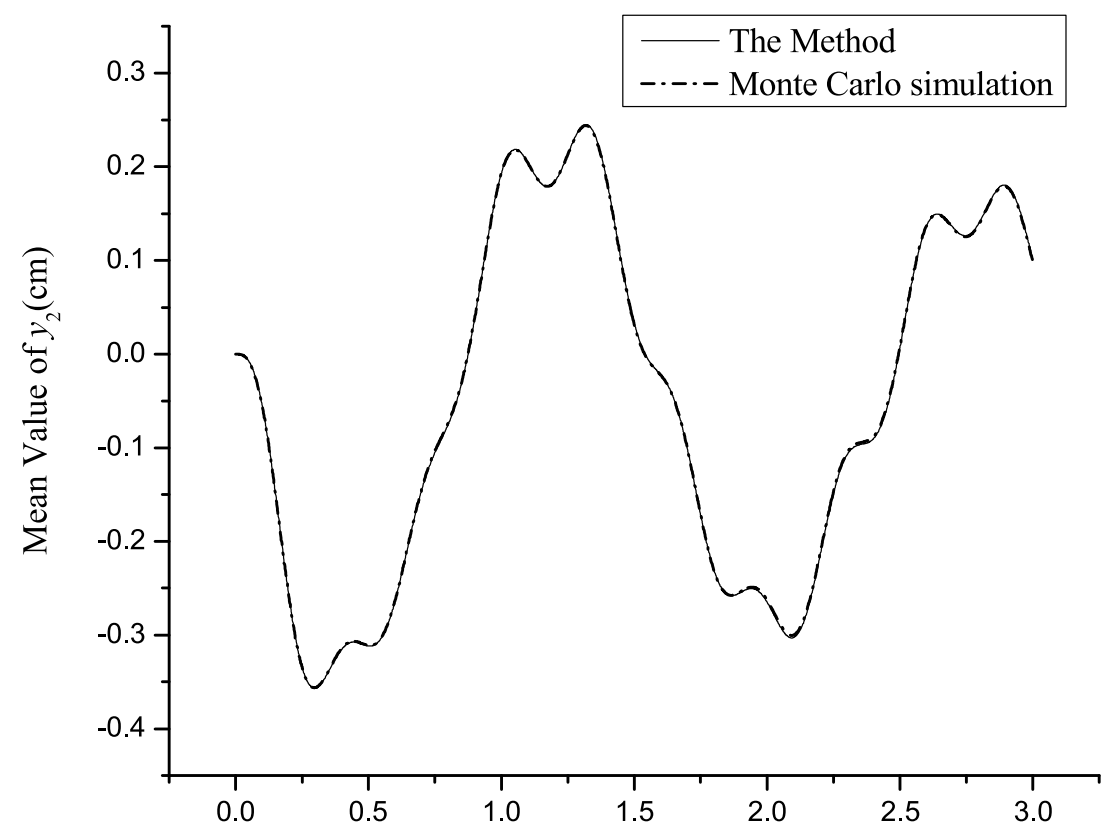

$t(\mathrm{~s})$

Fig. 3. The mean value curve of the displacement $y_{2}$.

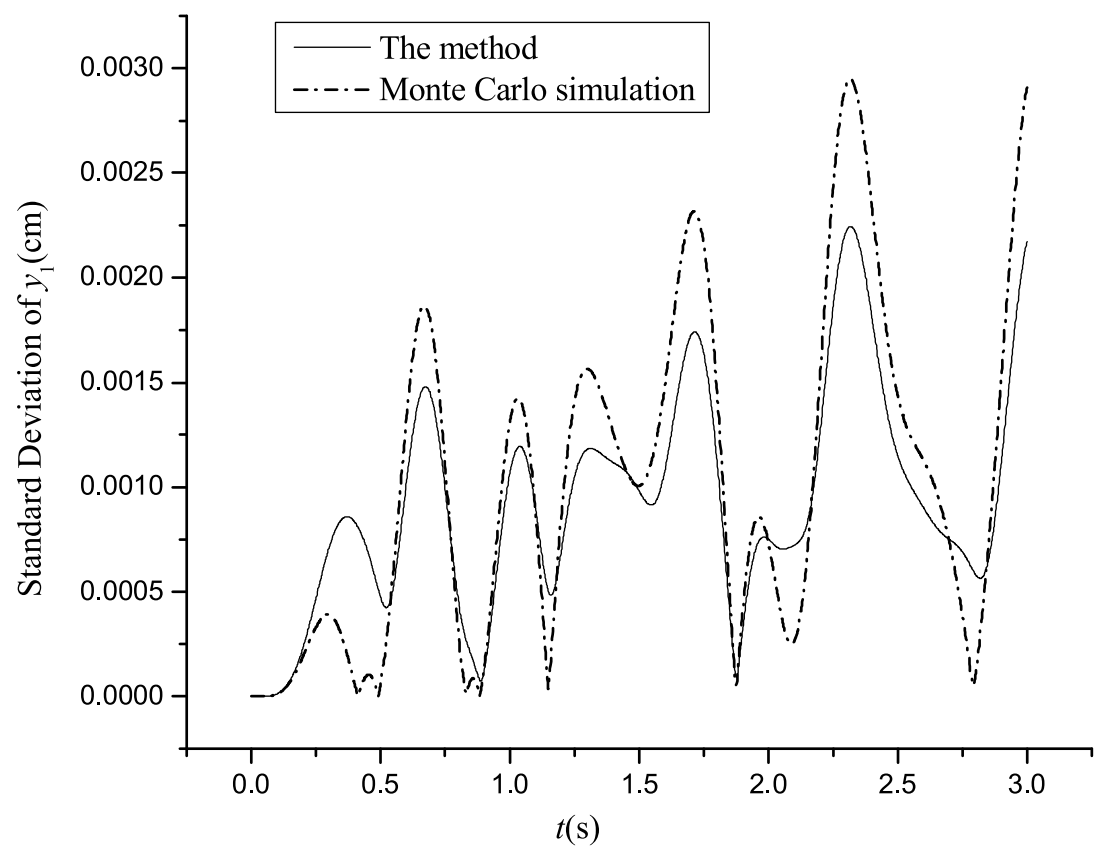

Fig. 4. The standard deviation curve of the displacement $y_{1}$

From Figs 2 to 6, the numerical results have demonstrated the accuracy of the method, which are compared with the Monte Carlo simulation results, and these results agree very well with the Monte Carlo simulation results. Various Monte Carlo simulation techniques are now available. In our analysis, the standard Monte Carlo method is used. The probability law of the vector-random variable is Gaussion, in the words, a normal random generator 


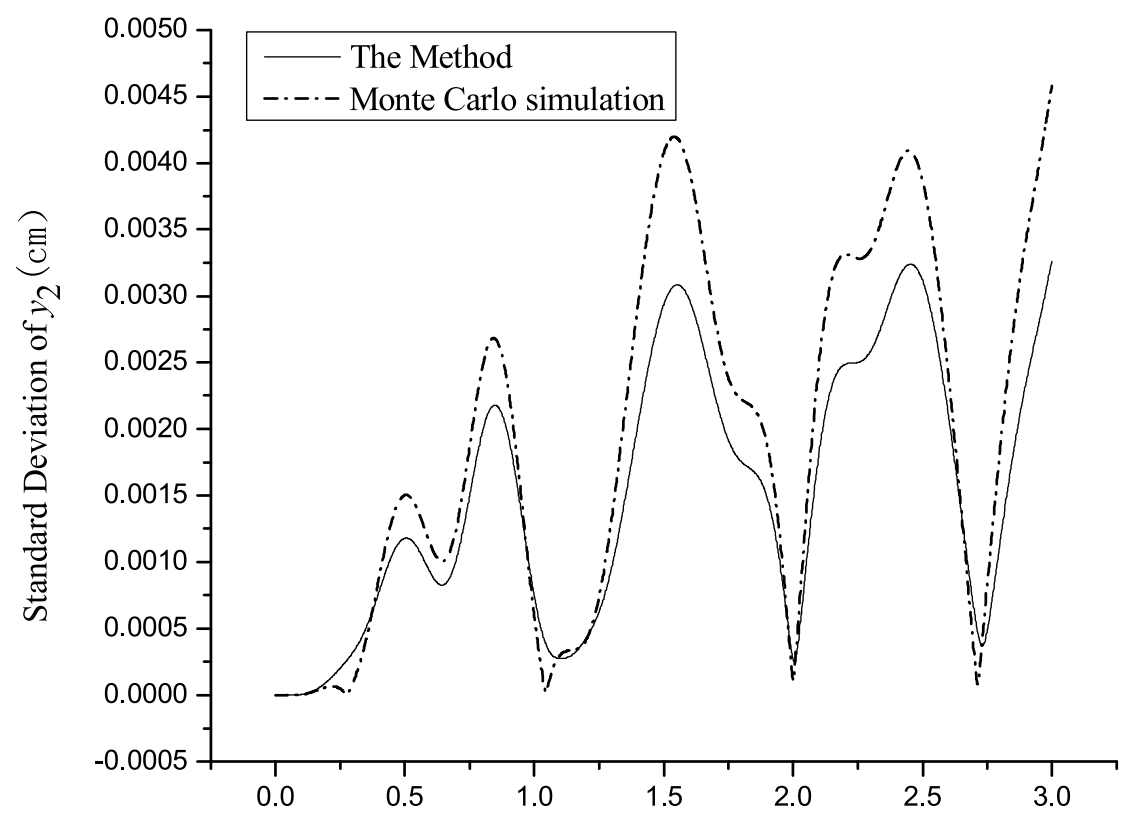

$t(\mathbf{s})$

Fig. 5. The standard deviation curve of the displacement $y_{2}$.

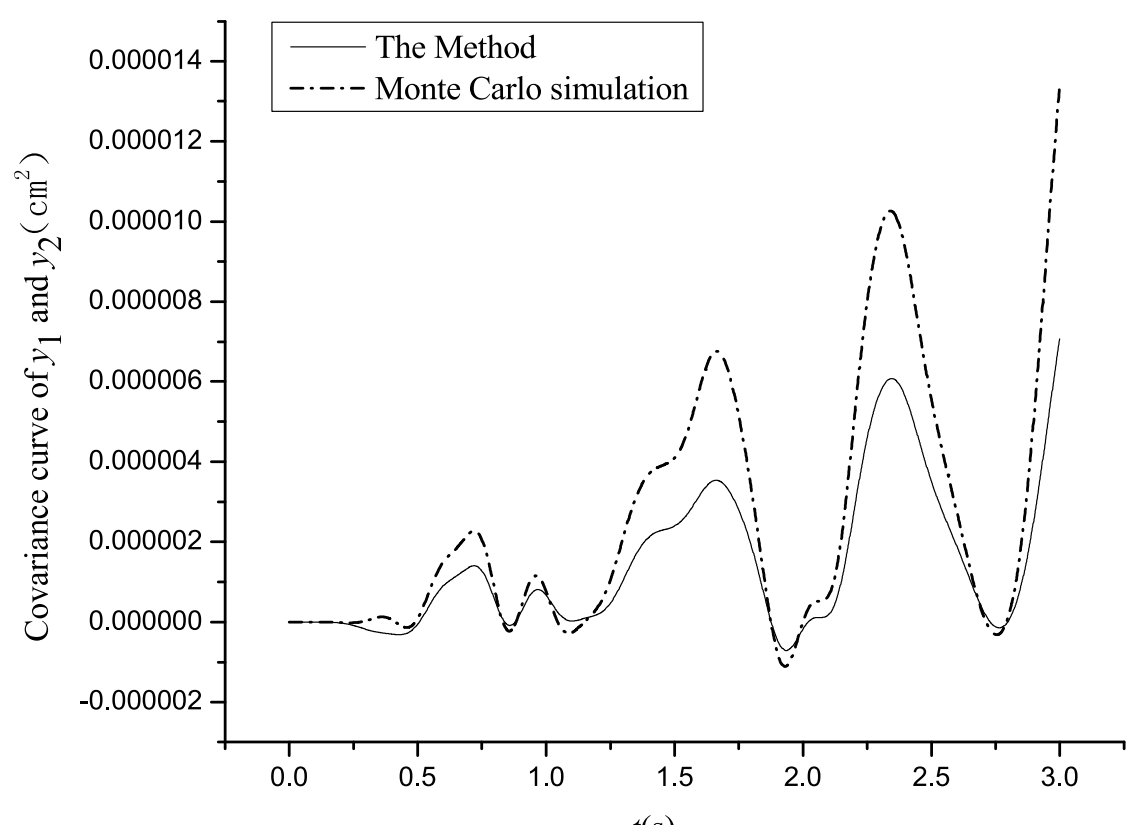

$t(\mathrm{~s})$

Fig. 6. The covariance curve of the $y_{1}$ and $y_{2}$.

is used, and the Monte Carlo simulation results are obtained with 1000 simulations. From this set of output, the probabilistic distribution properties, such as the mean and variance, are statistically estimated.

The purpose of this paper is to present a method for obtaining the response of stochastic hysteretic systems. The main advantage of the proposed method is that the stochastic hysteretic model is considered and the solution of 
multi-degree-of-freedom vibrations that is caused by stochastic hysteretic loop itself is obtained. The accuracy of the method is verified by Monte-Carlo simulations for all ranges of response levels.

\section{Conclusion}

This paper is based on the Bouc-Wen hysteretic model, the second moment method, and the perturbation technique to systematically develop dynamic response of random hysteretic system. The random response of random hysteretic system has been obtained. The results obtained are accorded with the Monte Carlo simulation results very well.

\section{Acknowledgements}

We would like to express our appreciation to Program for Changjiang Scholars and Innovative Research Team in University, Key National Science and Technology Special Project on "High-Grade CNC Machine Tools and Basic Manufacturing Equipments" (2010ZX04014-014), Chinese National Natural Science Foundation (50875039) and Key Projects in the National Science and Technology Pillar Program in the Eleventh Five-year Plan Period (2009BAG12A02).

\section{References}

[1] Y.K. Wen, Methods of random vibration for inelastic structures, Applied Mechanics Reviews 42(2) (1989), 1890-1905.

[2] T.K. Canghey, Random excitation of a system with bilinear hysteresis, Journal Applied Mechanics-Transactions of the ASME 27(4) (1960), 649-625.

[3] P.C. Jennings, Periodic response of a general yielding structure, Journal of the Engineering Mechanics Division-ASCE 90 (Apr. 1964), $131-165$.

[4] R. Bouc, Forced vibration of mechanical systems with hysteresis, Proceedings of the 4th Conference on Nonlinear Oscillation, Pargue, Czechoslovakia, 1967.

[5] Y.K. Wen, Method for random vibration of hysteretic systems, Journal of the Engineering Mechanics Division-ASCE 103(2) (1976), 249-203.

[6] E.H. Vanmarcke, Structural response to earthquakes, Seismic risk and engineering decision, Lonmitz and Rosenblueth, Eds. Elsevier, New York, 1976.

[7] W.D. Iwan, M.A. Moser and L.G. Pararizos, The stochastic response of strongly nonlinear systems with Coulomb damping elements, Proceedings IUTAM symposium on nonlinear stochastic dynamic engineering systems, Innsbruck, Austria, Jun, 1987, 455-466.

[8] W.D. Iwan, A generalization of the concept of equivalent linearization, International Journal of Nonlinear Mechanics 8(3) (1973), $279-287$.

[9] P.D. Spanos and W.D. Iwan, On the existence and uniqueness of solution generated by equivalent linearization, International Journal of Nonlinear Mechanics 13 (1978), 71-78.

[10] Y.K. Wen, Equivalent linearization for hysteretic systems under random excitation, Journal Applied Mechanics-Transactions of the ASME 47 (1) (1980), 150-154.

[11] B.F. Spencer and L.A. Bergman, On the reliability of a simple hysteretic system, Journal of the Engineering Mechanics Division-ASCE 111(12) (1985), 1502-1514.

[12] Y.K. Lin and G.Q. Cai, Probabilistic Structural Dynamics-Advanced Theory and Applications, McGraw-Hill, Inc., New York, 1995.

[13] M. Yar and J.K. Hammond, Parameter estimation for hysteretic systems, Journal of Sound and Vibration 117 (1987), 161-172.

[14] A.W. Smyth, S.F. Masri, A.G. Chassiakos and T.K. Caughey, On-line parametric identification of MDOF nonlinear hysteretic systems, Journal of Engineering Mechanics-ASCE 125(2) (1999), 133-142.

[15] Y.Q. Ni, J.M. Ko and C.W. Wong, Nonparametric identification of nonlinear hysteretic systems, Journal of Engineering Mechanics-ASCE 125(2) (1999), 206-215.

[16] R. Bouc and D. Boussaa, Drifting response of hysteretic oscillators to stochastic excitation, International Journal of Non-Linear Mechanics 37(8) (2002), 1397-1406.

[17] P.D. Spanos, P. Cacciola and G. Muscolino, Stochastic averaging of Preisach hysteretic systems, Journal of Engineering Mechanics-ASCE 130(11) (2004), 1257-1267.

[18] J. Guggenberger and H. Grundmann, Stochastic response of large FEM models with hysteretic behaviour in beam elements, Computer Methods In Applied Mechanics and Engineering 194(12-16) (2005), 1739-1756.

[19] S.F. Masri, R. Ghanem, F. Arrate et al., Stochastic nonparametric models of uncertain hysteretic oscillators, AIAA Journal 44(10) (2006), 2319-2330.

[20] Y.Wang, Z.G. Ying and W.Q. Zhu, Stochastic averaging of energy envelope of Preisach hysteretic systems, Journal of Sound and Vibration 321(3-5) (2009), 976-993.

[21] W.J. Vetter, Matrix calculus operations and Taylor expansions, SIAM Review 15(2) (1973), 352-369. 
[22] F. Ma, Extension of second moment analysis to vector-valued and matrix-valued functions, International Journal of Nonlinear Mechanics 22 (1987), 251-260.

[23] T.T. Baber and Y.K. Wen, Stochastic response of multistory yielding frames, Earthquake Engineering and Structural Dynamics 10 (1982), $403-416$. 

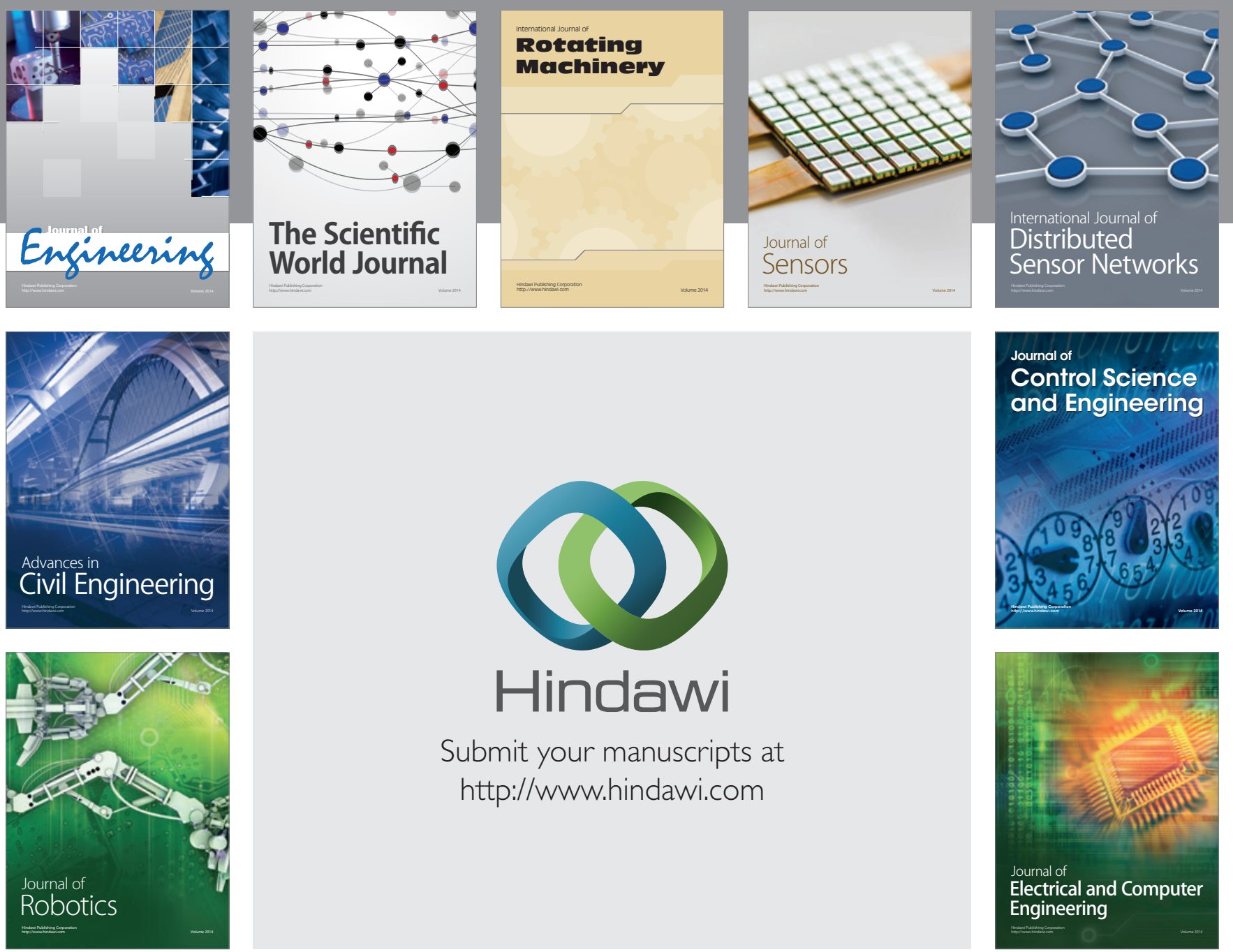

Submit your manuscripts at

http://www.hindawi.com
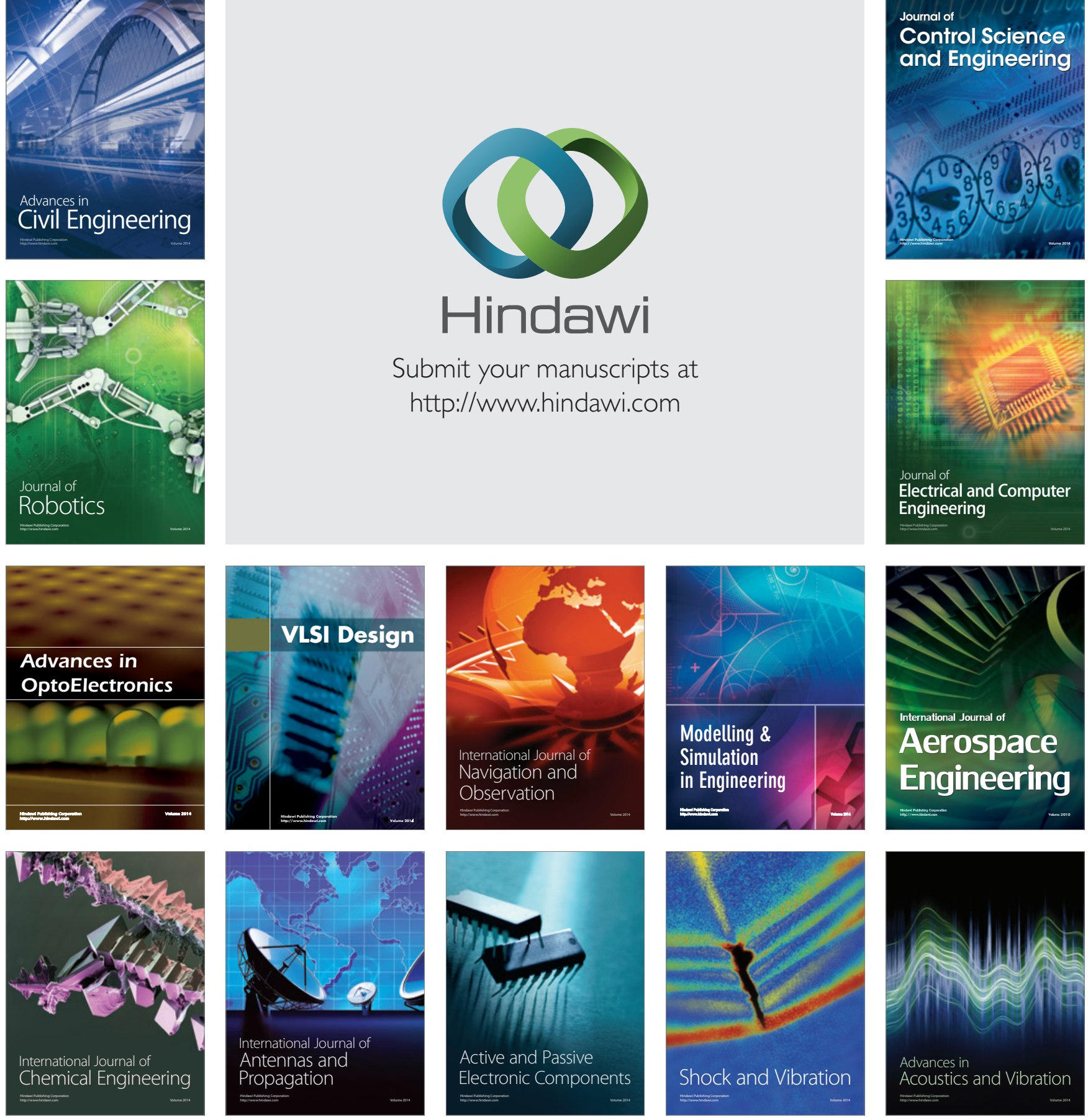